\title{
Sensibilidad y especificidad de la presión de pulso ampliada como prueba diagnóstica de IRC estadio K/DOQI III-b
}

\author{
Ana I. Pérez-Castañeda, ${ }^{1}$ Gilberto F. Vázquez-de Anda, ${ }^{\text {* }}$ Patricia Cerecero-Aguirre, ${ }^{1}$ \\ Rodolfo Rivas-Ruiz, ${ }^{2}$ María G. Delaye-Aguilar' y Juan O. Talavera ${ }^{3}$ \\ ${ }^{1}$ Universidad Autónoma del Estado de México, Clínica Multidisciplinaria de Salud, Estado de México; ${ }^{2}$ Instituto Mexicano del Seguro Social, Centro \\ Médico Nacional Siglo XXI, Centro de Adiestramiento en Investigación Clínica, Ciudad de México. ${ }^{3}$ Centro Médico ABC, Dirección de Enseñanza \\ e Investigación, Ciudad de México. México
}

\section{Resumen}

Introducción: La presión de pulso ampliada (PPA) se asocia a un filtrado glomerular calculado $\leq 60 / \mathrm{mL} / \mathrm{minuto} / 1.73 \mathrm{~m} \mathrm{~m}^{2}$, por lo que puede ser útil como prueba diagnóstica para identificar a personas con insuficiencia renal crónica (IRC) estadio K/DOQI III-b. Objetivo: Determinar la utilidad de la PPA como prueba diagnóstica de IRC estadio K/DOQI III-b. Método: Estudio de prueba diagnóstica que incluyó a pacientes adultos sin comorbilidades, registrados en la Cohorte de Trabajadores de la Salud. Se utilizó la fórmula CKD-EPI para calcular la filtración glomerular. Se determinó la presión de pulso restando la presión arterial diastólica a la presión arterial sistólica. Se calculó sensibilidad, especificidad, valor predictivo positivo, valor predictivo negativo y prevalencia. Se elaboró una curva ROC para determinar el área bajo la curva. Resultados: Se incluyeron 6215 pacientes. Se observó que una PPA $\geq 50 \mathrm{~mm} \mathrm{Hg}$ tuvo sensibilidad de $74 \%$, especificidad de $70 \%$, valor predictivo positivo de $1 \%$, valor predictivo negativo de $100 \%$ y prevalencia de $1 \%$. El punto de inflexión en la curva ROC para identificar IRC $K / D O Q I I I I-b$ fue de 0.71. Conclusión: $\mathrm{La} P P A \geq 50 \mathrm{~mm} \mathrm{Hg}$ es útil como prueba diagnóstica para identificar a personas con IRC estadio K/DOQI III-b.

PALABRAS CLAVE: Presión de pulso. Prueba diagnóstica. Filtración glomerular. K/DOQI III-b.

\section{Sensitivity and specificity of increased pulse pressure as a diagnostic test for K/DOQI III-b}

\begin{abstract}
Introduction: Increased pulse pressure (IPP) is associated an estimated glomerular filtration $\leq 60 / \mathrm{mL} / \mathrm{min} / 1.73 \mathrm{~m}$; thus, it can be useful as a diagnostic test to identify people with K/DOQI stage III-b chronic kidney disease (CKD). Objective: To determine the usefulness of IPP as a diagnostic test for K/DOQI stage III-b CKD. Method: Diagnostic test study that included adult patients without comorbidities, registered in the Health Workers Cohort. The CKD-EPI formula was used to calculate glomerular filtration. Pulse pressure was determined by subtracting diastolic from systolic blood pressure. Sensitivity, specificity, positive predictive value, negative predictive value and prevalence were calculated using standard formulas. A ROC curve was generated to determine the area under the curve. Results: A total of 6,215 patients were included. An IPP $\geq 50 \mathrm{mmHg}$ was observed to have a sensitivity of $74 \%$, specificity of $70 \%$, positive predictive value of $1 \%$, negative predictive value of $100 \%$ and a prevalence of $1 \%$. The inflection point in the ROC curve to identify K/DOQI III-b CKD was 0.71. Conclusion: An IPP $\geq 50 \mathrm{mmHg}$ is useful as a diagnostic test to identify people with K/DOQI stage III-b CKD.
\end{abstract}

KEY WORDS: Pulse pressure. Diagnostic test. Glomerular filtration. K/DOQI III-b.

Correspondencia:

*Gilberto F. Vázquez-de Anda

E-mail: gf_vazquez@ hotmail.com
Gac Med Mex. 2020;156:432-437

Disponible en PubMed

www.gacetamedicademexico.com

0016-3813/@ 2020 Academia Nacional de Medicina de México, A.C. Publicado por Permanyer. Este es un artículo open access bajo la licencia CC BY-NC-ND (http://creativecommons.org/licenses/by-nc-nd/4.0/). 


\section{Introducción}

La insuficiencia renal crónica (IRC) es una enfermedad que en etapas avanzadas incrementa el riesgo de muerte por enfermedad cardiovascular..$^{1-3} \mathrm{Si}$ se detecta en estadios tempranos se puede iniciar un tratamiento oportuno para conservar la función renal y retrasar el inicio de la terapia de sustitución mediante diálisis o hemodiálisis, hasta ahora el único tratamiento efectivo. ${ }^{1-5}$ Algunos pacientes pueden cursar las primeras etapas de la enfermedad asintomáticamente y debido a la accesibilidad limitada a los servicios de salud ${ }^{1,3}$ por lo general la IRC se diagnostica en etapas avanzadas, cuando están presentes las complicaciones tardías de la enfermedad con indicación de tratamiento sustitutivo, lo que incrementa el riesgo de muerte. ${ }^{1,6}$

Estudios recientes han identificado a la presión de pulso ampliada (PPA) como un factor de predicción de daño orgánico y riesgo cardiovascular, ${ }^{6-8}$ presente en los pacientes con IRC. Una PPA $\geq 45 \mathrm{~mm} \mathrm{Hg}^{6}$ se asocia a la disminución de la filtración glomerular (FG), independientemente de la edad, sexo, índice de masa corporal y presencia de diabetes. ${ }^{7.8}$ Sin embargo, Odaira et al. ${ }^{9}$ no encontraron asociación entre la IRC y la onda de reflexión del pulso por ultrasonido Doppler, lo que pone en duda la utilidad de la presión de pulso como indicador del índice de filtración glomerular (IFG). ${ }^{9}$

Actualmente, para estadificar a la IRC se utiliza la escala de K/DOQI (Kidney Disease Outcomes Quality Initiative), ${ }^{1}$ con base en el IFG, el cual puede calcularse (IFGc) a partir de la escala CKD-EPI (Chronic Kidney Disease Epidemiology Collaboration Equation), la cual incluye edad, creatinina sérica, sexo y raza. La escala identifica cinco estadios de Ia IRC, de los cuales el estadio III-b (30 a $45 \mathrm{~mL} /$ minuto $/ 1.73 \mathrm{~m}^{2}$ ) se asocia a muerte por enfermedad cardiovascular. El estadio K/DOQI III-b puede corresponder a una población objetivo en la práctica clínica para identificar tempranamente a pacientes con IRC. La asociación entre la PPA y la disminución del IFGc puede tener un valor diagnóstico para esta población objetivo e identificarla tempranamente para ofrecer un tratamiento oportuno.

Por lo anterior, y dada la importancia de desarrollar nuevos métodos diagnósticos de escrutinio que permitan identificar precozmente la enfermedad renal, el objetivo de nuestro estudio fue determinar la utilidad de la PPA para identificar a pacientes con IRC estadio K/DOQI III-b.

\section{Método}

El presente es un estudio de prueba diagnóstica. Para la selección y descripción de los participantes se revisó la información obtenida a través de una muestra no probabilística seleccionada a conveniencia de los sujetos adultos y sin comorbilidades que participaron en el estudio mexicano de la Cohorte de Trabajadores de la Salud, integrada por empleados y familiares de trabajadores del Instituto Mexicano del Seguro Social del Estado de Morelos, el Instituto Nacional de Salud Pública y la Universidad Autónoma del Estado de México. Antes del inicio del estudio, el protocolo fue revisado y aprobado por los comités de ética e investigación de las instituciones participantes.

Se invitó a 13275 sujetos a colaborar en la Cohorte de Trabajadores de la Salud entre marzo de 2003 y abril de 2006. Se elaboró un registro para los 10192 sujetos que participaron. Se revisó la información de 9142 pacientes cuyos datos para la realización del estudio estuvieran completos. Se excluyeron los datos de los pacientes < 18 años y con condiciones que alteran la presión de pulso, como diabetes, hipertensión y cardiopatías, entre otras. Finalmente, se analizaron los datos de 6215 sujetos adultos sin comorbilidades (Figura 1). Se incluyeron los datos bioquímicos y antropométricos de 6215 sujetos adultos sin comorbilidades contenidos en la base de datos de la Cohorte de Trabajadores de la Salud.

El peso de los pacientes se determinó con una báscula electrónica calibrada (modelo BC-533, Tanita); los participantes fueron pesados descalzos y con una cantidad mínima de ropa. La estatura se midió con un estadiómetro convencional (Seca), con el paciente de pie y descalzo, erguido, con los hombros abajo y la cabeza en posición para mantener el plano de Frankfurt. Las mediciones se tomaron con la cinta en un plano horizontal perpendicular a la escala vertical, tocando la parte superior de la cabeza en el momento de la inspiración.

Se midió la presión arterial mientras los participantes estaban sentados con su brazo apoyado sobre una superficie plana y a nivel del corazón; se empleó un baumanómetro con monitor automático digital marca Citizen. Enfermeras entrenadas para llevar a cabo la obtención de la presión arterial realizaron tres mediciones en cada individuo. Mediante coeficientes de concordancia se evaluó la reproducibilidad de mediciones antropométricas y clínicas, por lo que se determinó una concordancia de 0.83 a 0.90 . 


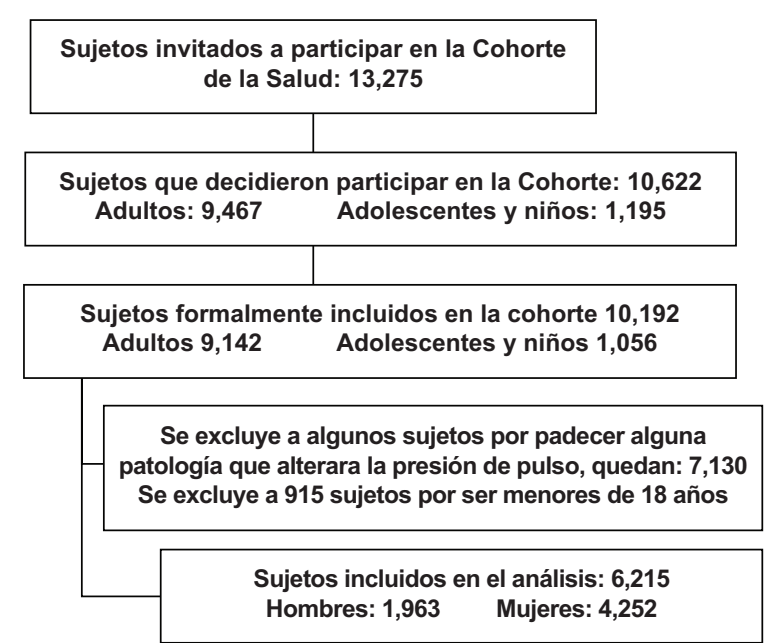

Figura 1. Criterios utilizados para la selección de la muestra poblacional estudiada.

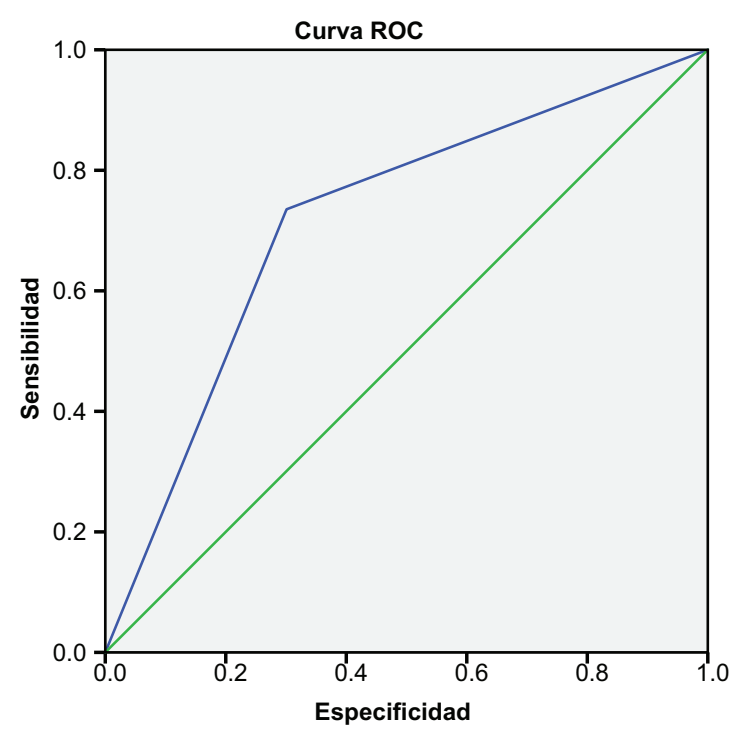

Los segmentos de diagonal se generan mediante empates

Figura 2. Curva ROC correspondiente a una presión de pulso ampliada $>50 \mathrm{~mm} \mathrm{Hg}$ y un filtrado glomerular $<45 \mathrm{~mL} /$ minuto/1.73 $\mathrm{m}^{2}$.

Se obtuvieron $20 \mathrm{~mL}$ de sangre por medio de punción venosa periférica, para lo cual se pidió a los sujetos que se presentaran con más de ocho horas de ayuno antes de la toma de la muestra. Posteriormente, todas las muestras se analizaron en el mismo laboratorio mediante un equipo Selectra XL Instrument ${ }^{\circledR}$ (Randox Laboratories, Antrim, Reino Unido) y de acuerdo con el procedimiento establecido por la Federación Internacional de Química y Laboratorio Médico.
La PPA se obtuvo mediante la diferencia de la presión arterial sistólica menos la presión arterial diastólica. Considerando que $40 \mathrm{~mm} \mathrm{Hg}$ es un parámetro normal de la presión de pulso, se determinó que al elevarse por lo menos $5 \mathrm{~mm} \mathrm{Hg}$ sería denominada PPA. Para el presente estudio se analizaron tres categorías:6-8

- Incremento de 5 a $9 \mathrm{~mm} \mathrm{Hg}$ ( $\geq 45 \mathrm{a} \leq 49 \mathrm{~mm} \mathrm{Hg}$ ).

- Incremento de 10 a $14 \mathrm{~mm} \mathrm{Hg}(\geq 50$ a $\leq 54 \mathrm{~mm} \mathrm{Hg}$ ).

- Incremento superior a los $15 \mathrm{~mm} \mathrm{Hg}$ ( $\geq 55 \mathrm{~mm} \mathrm{Hg}$ ).

Finalmente, el índice de filtración glomerular calculado (IFGc) se determinó con la ecuación formulada por Chronic Kidney Disease Epidemiology Collaboration o CKD-EPI ${ }^{10}$ la cual se expresa en $\mathrm{mL} /$ minuto/1.73 $\mathrm{m}^{2}$ y se ajusta en cada individuo por raza (blanca, negra u otras), edad, sexo y niveles de creatinina sérica. Finalmente, la velocidad de la filtración glomerular se clasificó según las guías internacionales K/DOQI (Kidney Disease Outcomes Quality Initiative) y se analizaron los datos de los pacientes con una filtración glomerular $\leq 60 \mathrm{~mL} /$ minuto/1.73 $\mathrm{m}^{2}$.,11,12

Se utilizó el programa de Excel versión 2010 y el programa estadístico SPSS versión 21 para el análisis estadístico.

Se elaboraron tablas $2 \times 2$ para contrastar los datos del IFGc y la presión de pulso de los pacientes. Para la prueba diagnóstica se determinó la sensibilidad, especificidad, valor predictivo positivo (VPP), valor predictivo negativo (VPN), prevalencia, certeza diagnóstica y razón de verosimilitud de la prueba, mediante fórmulas convencionales. ${ }^{13-15}$

Se elaboró una curva ROC (receiver operator characteristic) para determinar el punto de corte óptimo para detectar disminución de la $F G$ a través de la ampliación de la presión de pulso. ${ }^{14}$

\section{Consideraciones bioéticas}

Antes de iniciar el estudio de la Cohorte de Trabajadores de la Salud, el protocolo fue revisado y aprobado por los comités de ética y de investigación de las instituciones participantes: el Instituto Mexicano del Seguro Social (12CEI 09006 14), el Instituto Nacional de Salud Pública (13CEI 17007 36) y la Universidad Autónoma del Estado de México (1233008X0236). Se obtuvo el consentimiento informado por escrito de todos los participantes antes de la inscripción al estudio. Se garantizó la confidencialidad de los datos biométricos de los participantes 
Tabla 1. Resumen de los análisis estadísticos de la PPA $\geq 45 \mathrm{~mm} \mathrm{Hg}$ con FG $\leq 60 \mathrm{~mL} /$ minuto/1.73 m² (K/DOQI III)

\begin{tabular}{|c|c|c|c|}
\hline & $\mathrm{PPA} \geq 45 \mathrm{~mm} \mathrm{Hg}$ & $P P A \geq 50 \mathrm{~mm} \mathrm{Hg}$ & $\mathrm{PPA} \geq 55 \mathrm{~mm} \mathrm{Hg}$ \\
\hline Sensibilidad (\%) & 65 & 48 & 31 \\
\hline Especificidad (\%) & 50 & 71 & 85 \\
\hline Valor predictivo positivo (\%) & 10 & 11 & 14 \\
\hline Valor predictivo negativo (\%) & 94 & 95 & 94 \\
\hline Prevalencia (\%) & 8 & 7 & 7 \\
\hline Certeza diagnóstica (\%) & 52 & 69 & 82 \\
\hline Área bajo la curva & 0.57 & 0.59 & 0.58 \\
\hline IC $95 \%$ & $0.55-0.60$ & $0.56-0.62$ & $0.55-0.61$ \\
\hline
\end{tabular}

PPA = presión de pulso ampliada, FG = filtración glomerular, IC = intervalo de confianza.

Tabla 2. Resumen de los análisis estadísticos para una PPA $\geq 45 \mathrm{~mm} \mathrm{Hg}$ con FG $\leq 45 \mathrm{~mL} / \mathrm{minuto} / .73 \mathrm{~m}^{2}$ (K/DOQI III-b)

\begin{tabular}{|l|c|c|c|}
\hline & PPA $\geq \mathbf{4 5 m ~ H g}$ & PPA $\geq 50$ mm Hg & PPA $\geq 55$ mm Hg \\
\hline Sensibilidad (\%) & 85 & 74 & 47 \\
\hline Especificidad (\%) & 49 & 70 & 2 \\
\hline Valor predictivo positivo (\%) & 1 & 1 & 100 \\
\hline Valor predictivo negativo (\%) & 100 & 100 & 1 \\
\hline Prevalencia (\%) & 1 & 1 & 84 \\
\hline Certeza diagnóstica (\%) & 50 & 70 & 0.65 \\
\hline Área bajo la curva & 0.67 & 0.71 & $0.55-0.76$
\end{tabular}

PPA = presión de pulso ampliada, FG = filtración glomerular, IC = intervalo de confianza

mediante la identificación de cada sujeto con un número de registro y no con su nombre.

El presente estudio cumplió con los criterios de investigación sin riesgo estipulados en el artículo 24 de la Declaración de Helsinki de la Asociación Médica Mundial, así como con el criterio del título segundo, capítulo 1, artículo 17, sección 1 del Reglamento de la Ley General de Salud en Materia de Investigación para la Salud. En cuanto a aspectos éticos de la investigación en seres humanos, se emplearon técnicas y métodos de investigación documental retrospectivos con los cuales no se realizó ninguna intervención intencionada (investigación sin riesgo); a pesar de ello, se obtuvo la carta de consentimiento informado que firmaron los participantes al integrarse a la cohorte, en la cual consintieron en contestar un cuestionario, proporcionar muestras de sangre y ser medidos antropométricamente.

\section{Resultados}

Se analizaron los datos de 6215 pacientes adultos sin comorbilidades incluidos en la Cohorte de Trabajadores de la Salud. La media de edad de los participantes fue de $41.3 \pm 13.6$ años, la presión sistólica fue de $115 \pm 12.9 \mathrm{~mm} \mathrm{Hg}$, la diastólica de $70.1 \pm 10.1 \mathrm{~mm} \mathrm{Hg}$, la de pulso de $45.3 \pm 9.8 \mathrm{~mm} \mathrm{Hg}$, la creatinina sérica de $0.9 \pm 0.15 \mathrm{mg} / \mathrm{dL}$ y la $\mathrm{FGc}$ de $85.9 \pm 20.3 \mathrm{~mL} /$ minuto $/ 1.73 \mathrm{~m}^{2}$.

En la Tabla 1 se describen los datos de sensibilidad, especificidad, valor predictivo positivo, valor predictivo negativo, prevalencia, certeza diagnóstica de los pacientes con una filtración glomerular $\leq 60 \mathrm{~mL} /$ minuto $/ 1.73 \mathrm{~m}^{2}$ (K/DOQI III) y PPA $\geq 45 \mathrm{~mm} \mathrm{Hg}$. En la Tabla 2 se encuentran los valores de la prueba diagnóstica calculados para PPA $\geq 45 \mathrm{~mm} \mathrm{Hg}$ y FGc $\leq 45 \mathrm{~mL} /$ minuto $/ 1.73 \mathrm{~m}^{2}$, correspondiente a IRC estadio K/DOQI III-b. 
El área bajo la curva reflejada en las tablas y los puntos de corte para las pruebas fueron determinados mediante la elaboración de curvas ROC. La Figura 2 muestra la línea de discriminación de la prueba de acuerdo con los valores cruzados de presión de pulso $\geq 50 \mathrm{~mm} \mathrm{Hg}$ y FGc $\geq 30 \mathrm{y} \leq 45 \mathrm{~mL} /$ minuto $/ 1.73 \mathrm{~m}^{2}$ (K/DOQI III-b).

\section{Discusión}

Nuestro estudio demuestra que la presión de pulso $\geq 50 \mathrm{~mm} \mathrm{Hg}$ tiene una capacidad diagnóstica aceptable para identificar a pacientes sin morbilidades asociadas, con IRC en estadio KDOQI III-b. Según el Instituto Mexicano del Seguro Social, la IRC en etapas avanzadas de la enfermedad se encuentra entre las primeras 10 causas de mortalidad general y representa la octava causa de defunción en el varón en edad productiva y la sexta en la mujer de 20 a 59 años. ${ }^{3,4}$ Debido a lo anterior, urge identificar tempranamente a pacientes con riesgo de disminución de la filtración glomerular en estadios en los que la enfermedad es asintomática.

En nuestro estudio se observó que la media de la presión de pulso de la población analizada se encontraba $5 \mathrm{~mm} \mathrm{Hg}$ sobre los valores de referencia (40 mm Hg). De acuerdo con la literatura, esta elevación es un factor de iniciación y progresión a K/DOQI III a largo plazo, ${ }^{6-8}$ debido a que conforme aumenta la presión de pulso, se incrementa la velocidad de progresión de la enfermedad renal. ${ }^{11}$

La edad de los sujetos de estudio no constituyó un factor de riesgo para IRC; sin embargo, debido a que la filtración glomerular disminuye $1 \mathrm{~mL} /$ minuto/año a partir de los 30 años, se recomienda monitorear la función renal por lo menos una vez al año en los pacientes adultos. Actualmente se considera como prueba de escrutinio el valor de la creatinina sérica o el cálculo del IFG mediante fórmulas convencionales. ${ }^{4,11}$

Nuestro estudio demuestra (a través de la curva $\mathrm{ROC}$ ) que la prueba tiene una capacidad para identificar a dos tercios de pacientes con PPA $\geq 50 \mathrm{~mm} \mathrm{Hg}$ y disminución del IFGc equivalente a IRC estadio K/DOQI III-b. .,6-8,10-12,14

La capacidad de la presión de pulso para descartar disminución del IFGc equivalente a estadio KDOQI III-b (VPN), cuando el paciente presenta presión de pulso $\leq 50 \mathrm{~mm} \mathrm{Hg}$ es elevada.12,13,15 El cálculo de los valores predictivos para determinar la probabilidad de desarrollar la enfermedad renal depende de la prevalencia de la población de estudio; por este motivo, el
VPP es bajo, porque la prevalencia de la enfermedad en nuestra población es baja, mientras que el VPN aumenta. $12,13,15$

Nuestro estudio determinó que la prevalencia y el valor predictivo positivo (VPP) fueron bajos, por lo que la prueba detectaría IRC estadio K/DOQI III-b en uno de cada 100 pacientes mediante una técnica rápida, no invasiva y que se realiza en cada visita al médico o institución de salud. 12,13,15 Dado que el VPN obtenido es alto, la prueba es capaz de descartar la enfermedad en un paciente con sospecha de IRC estadio K/DOQI III-b.

Por otra parte, sabemos que $70 \%$ de las veces que se realice la prueba en un individuo, el diagnóstico será acertado y que si el paciente tiene una PPA $\geq 50 \mathrm{~mm} \mathrm{Hg}$, su FG será menor en comparación con las personas sanas, por lo que la PPA $\geq 50 \mathrm{~mm} \mathrm{Hg}$ puede utilizarse como prueba diagnóstica de escrutinio para IRC estadio K/DOQI III-b. ${ }^{12-15}$

La PPA por sí misma es una característica presente en los pacientes con K/DOQIII-b, es un predictor de daño orgánico y un factor de riesgo cardiovascular. ${ }^{6,8}$ La particularidad de la PPA es su asociación a complicaciones y muerte por enfermedad cardiovascular. ${ }^{16-18} \mathrm{~A}$ pesar de la evidencia, Mari Odaira y Colin D. Chue, con sus respectivos colaboradores, ${ }^{9,19}$ afirman que aunque la PPA puede estar presente en pacientes con $\mathrm{FG}<60 / \mathrm{mL} /$ minuto $/ 1.73 \mathrm{~m}^{2}$, en modelos de regresión lineal ajustados por edad, sexo y algunas comorbilidades no se observó asociación entre la PPA y la disminución de la FG, por lo que la PPA no podría utilizarse como un indicador de FG. ${ }^{9,19}$ Sin embargo, la ampliación de la PP $\geq 5 \mathrm{~mm} \mathrm{Hg}$ por encima de la normalidad ${ }^{6}$ se encuentra asociada a IRC por la disminución del FGc. Por lo anterior, la PPA puede utilizarse como un marcador clínico para identificar a pacientes con IRC según las guías K/DOQI. ${ }^{1}$

El tratamiento sustitutivo de la función renal tiene un costo elevado, que no puede ser cubierto por el paciente de no contar con acceso a la seguridad social, por lo que la situación económica familiar y la calidad de vida del paciente se ven afectadas al presentarse la IRC. ${ }^{1,3}$ De ahí la importancia de la detección oportuna de la disminución de la FG mediante pruebas diagnósticas de escrutinio sensibles, específicas, de bajo costo y fáciles de implementar, como la PPA, ya que en etapas avanzadas de la enfermedad renal (K/DOQI IV-V) aumenta la probabilidad de que el paciente padezca complicaciones secundarias a la enfermedad o al tratamiento sustitutivo tardío., 1,3,8

De acuerdo con los resultados obtenidos, se planea realizar nuevos estudios en los que se incluyan los 
pacientes con comorbilidades para determinar nuevos puntos de corte, así como los datos más recientes de los pacientes de la Cohorte de Trabajadores de la Salud.

Al analizar los datos se identificaron algunas limitaciones: debimos excluir a 2927 individuos porque la información no se encontraba completa. La principal limitación del estudio consistió en que solo se incluyeron datos de personas que viven en el Estado de México y Morelos, por lo cual los resultados no pueden generalizarse al resto de la población mexicana.

Finalmente, nuestro estudio demuestra que los pacientes que presentaron una PPA $\geq 50 \mathrm{~mm} \mathrm{Hg}$ tuvieron un $\mathrm{FG} \leq 45 / \mathrm{mL} /$ minuto/1.73 $\mathrm{m}^{2}$, por lo que la PPA $\geq 50 \mathrm{~mm} \mathrm{Hg}$ puede utilizarse como prueba diagnóstica de escrutinio oportuna, sensible y específica para IRC estadio K/DOQI III-b.

\section{Conclusión}

La PPA $\geq 50 \mathrm{~mm} \mathrm{Hg}$ puede utilizarse como prueba diagnóstica de escrutinio, sensible y específica para la detección oportuna de IRC estadio K/DOQI III-b en adultos sin comorbilidades.

\section{Conflicto de Intereses}

Ninguno.

\section{Financiamiento}

Ana Ivonne Pérez Castañeda recibió beca para estudios de doctorado por parte del Consejo Nacional de Ciencia y Tecnología de México.

\section{Responsabilidades éticas}

Protección de personas y animales. Los autores declaran que para esta investigación no se realizaron experimentos en seres humanos ni en animales.

Confidencialidad de los datos. Los autores declaran que siguieron los protocolos de su centro de trabajo en cuanto a la publicación de datos de pacientes.
Derecho a la privacidad y consentimiento informado. Los autores declaran que en este artículo no aparecen datos de pacientes.

\section{Bibliografía}

1. Sarcona E, Díaz M. Effect of renal disease on ambulatory blood pressure monitoring. Rev Argent Cardiol. 2015;83:135-139.

2. Medeiros M, Andrade-Veneros GD, Toussaint-Martínez-de Castro G, Ortiz-Vásquez L, Hernández-Sánchez AM, Olvera N, et al. Prevalencia de enfermedad renal en niños aparentemente sanos con antecedente familiar de terapia de reemplazo renal. Bol Med Hosp Infant Mex. 2015;72:257-261.

3. Méndez-Durán A, Méndez-Bueno JF, Tapia-Yáñez T, Muñoz-Montes A, Aguilar-Sánchez L. Epidemiología de la insuficiencia renal crónica en México. Dial Transplant. 2014;35:148-156.

4. Méndez-Durán A, Pérez-Aguilar G, Ayala-Ayala F, Ruiz-Rosas RA, González-Izquierdo JJ, Dávila-Torres. Panorama epidemiológico de la insuficiencia renal crónica en el segundo nivel de atención del Instituto Mexicano del Seguro Social. Dial Transplant. 2014;35:148-156.

5. Amato D, Álvarez-Aguilar C, Castañeda-Limones R, Rodriguez E, Vila-Diaz M, Arreola F, et al. Prevalence of chronic kidney disease in an urban Mexican population. Kidney Int Suppl. 2005,97:S11-S17.

6. Peralta CA, Jacobs DR, Katz R, Ix JH, Madero M, Duprez DA, et al. Association of pulse pressure, arterial elasticity, and endothelial function with kidney function decline among adults with estimated GFR $>60 \mathrm{~mL} /$ $\mathrm{min} / 1.73 \mathrm{~m}^{2}$ : the Multi-Ethnic Study of Atherosclerosis (MESA). Am J Kidney Dis. 2012:59:41-49.

7. Safar ME, PlanteGE, Mimran A. Arterial stiffness, pulse pressure, and the kidney. Am J Hypertens. 2015;8:561-569.

8. Luño J, Gómez-Campderá FJ, García-de Vinuesa S, Goicoechea M. Factores determinantes de la presión de pulso en la enfermedad renal crónica. Nefrologia (Madr). 2004;24:29-32.

9. Odaira M, Tomiyama H, Matsumoto C, Yamada J, Yoshida M, Shiina K, et al. Association of serum cystatin $\mathrm{C}$ with pulse wave velocity, but not pressure wave reflection, in subjects with normal renal function or mild chronic kidney disease. Am J Hypertens. 2010;23:967-973.

10. Wood AJ, Churilov L, Perera N, Thomas D, Poon A, Maclsaac RJ, Jerums G, Ekinci El. Estimating glomerular filtration rate: performance of the CKD-EPI equation over time in patients with type 2 diabetes. J Diabetes Complications. 2016;30:49-54.

11. Flores JC, Alvo M, Borja H, Morales J, Vega J, Zúñiga $C$, et al. Enfermedad renal crónica: clasificación, identificación, manejo y complicaciones. Rev Med. 2009;137:137-177.

12. van Stralen KJ, Stel VS, Reitsma JB, Dekker FW, Zoccali C, Jager KJ. Diagnostic methods I: sensitivity, specificity, and other measures of accuracy. Kidney Int. 2009;75:1257-1263.

13. Talavera JO, Wacher-Rodarte NH, Rivas-Ruiz R. Investigación clínica II. Estudios de proceso (prueba diagnóstica). Rev Med Inst Mex Seguro Soc. 2011;49:163-170.

14. Tripepi G, Jager KJ, Dekker FW, Zoccali C, Diagnostic methods 2: receiver operating characteristic (ROC) curves. Kidney Int. 2009;76: 252-256.

15. Burgos ME, Manterola C. Cómo interpretar un artículo sobre pruebas diagnósticas. Rev Chil Cir. 2010;62:301-308.

16. Briet M, Maruani G, Collin C, Bozec E, Gauci C, Boutouyrie P, et al. Age-independent association between arterial and bone remodeling in mild-to-moderate chronic kidney disease. Nephrol Dial Transplant. 2010:25:191-197.

17. DeLoach SS, Appel LJ, Chen J, Joffe MM, Gadegbeku CA, Mohler ER, et al. Aortic pulse pressure is associated with carotid IMT in chronic kidney disease: report from chronic renal insufficiency cohort. Am J Hypertens. 2009;22:1235-1241.

18. Verbeke F, van Biesen W, Peeters P, van Bortel LM, Vanholder RC. Arterial stiffness and wave reflections in renal transplant recipients. Nephrol Dial Transplant. 2007:22:3021-3027

19. Chue CD, Edwards NC, Davis LJ, Steeds RP, Townend JN, Ferro CJ. Serum phosphate but not pulse wave velocity predicts decline in renal function in patients with early chronic kidney disease. Nephrol Dial Transplant. 2011;26:2576-2582. 